\title{
Article
}

\section{Borderline personality traits, rumination and self-injurious behavior: An empirical test of the emotional cascades model in adult male offenders}

Gardner, Kathryn Jane, Dodsworth, Jo and Selby, Edward

Available at http://clok.uclan.ac.uk/12166/

Gardner, Kathryn Jane ORCID: 0000-0003-3904-1638, Dodsworth, Jo and Selby, Edward (2014) Borderline personality traits, rumination and selfinjurious behavior: An empirical test of the emotional cascades model in adult male offenders. Journal of Forensic Psychology Practice, 14 (5). pp. 398-417. ISSN 1522-8932

It is advisable to refer to the publisher's version if you intend to cite from the work. http://dx.doi.org/10.1080/15228932.2014.962379

For more information about UCLan's research in this area go to http://www.uclan.ac.uk/researchgroups/ and search for <name of research Group>.

For information about Research generally at UCLan please go to http://www.uclan.ac.uk/research/

All outputs in CLoK are protected by Intellectual Property Rights law, including Copyright law. Copyright, IPR and Moral Rights for the works on this site are retained by the individual authors and/or other copyright owners. Terms and conditions for use of this material are defined in the policies page. 


\begin{abstract}
The aim of the study was to examine whether the emotional cascade model, which suggests that the relationship between emotional and behavioural dysregulation in Borderline Personality Disorder (BPD) is mediated by rumination, applies to a sample of adult male offenders. The study focused on individuals with "BPD traits” and two dysregulated behaviors that are particularly relevant to the prison population: non-suicidal self-injury (NSSI) and suicidality. Participants were 179 adult male offenders detained in a mediumsecure prison, all of whom completed a battery of measures and self-reported their actual prison behaviour. The results support the application of the emotional cascade model to adult male offenders, suggesting that emotional cascades may play an important role in NSSI and suicidality in this population, although this relationship needs further exploration. These findings highlight the benefits of targeting rumination to manage risk of NSSI and suicidality within custodial settings.
\end{abstract}

Keywords:

Borderline Personality Disorder; emotional cascades; rumination; non-suicidal self-injury; suicidal behaviour; suicidality; male offenders 
Borderline personality disorder, rumination and self-injurious behaviour: An empirical test of the emotional cascades model in adult male offenders

Borderline Personality Disorder (BPD) is characterised by chaotic interpersonal relationships, affective instability, poor anger control, identity diffusion, impulsive behaviour, chronic feelings of emptiness, and self-injurious and suicidal behaviour (APA, 1995). BPD has been conceptualized primarily as a disorder of emotion dysregulation (Linehan, 1993) and there is much empirical evidence to support this (e.g. Bornovalova et al., 2008; Conklin, Bradley \& Westen, 2006). BPD or BPD traits are also linked to dysregulated behaviours such as non-suicidal self-injury (NSSI) (e.g. Klonsky, Oltmanns \& Turkheimer, 2003), suicide (e.g. Black et al., 2007), substance abuse (e.g. Trull, Sher, Minks-Brown, Durbin \& Burr, 2000), aggression (Gardner, Archer \& Jackson, 2012) and binge eating (e.g. Sansone, Wiederman \& Sansone, 2000). Theories of BPD suggest that emotional dysregulation is the driving force behind these behaviours (Linehan, 1993). While evidence on this topic is accumulating within clinical and community settings, less is known about how this it manifests within prison settings.

The prevalence of BPD in the general population is typically around 1-2\% (APA, 1995; Grant et al., 2008; Samuels et al., 2002), but much empirical evidence has shown that BPD is overrepresented in prison populations. In a summary of several studies Sansone and Sansone (2009) found a prevalence of between 25-50\% within prison populations. There are gender effects though, with the percentage of women meeting BPD criteria within prison being typically ten times that of men (Hawton, Linsell, Adeniji, Sariaslan, \& Fazel, 2014). However, the prevalence of BPD in males is still inflated and of higher prevalence than in the general population (Leue, Borchard \& Hoyer, 2004). It is therefore a significant cause for 
concern within the prison environment with males with BPD likely accounting for a number of the problems seen in forensic settings (e.g., aggression, self-injury).

Dysregulated behaviours such as aggression and self-injury that are associated with BPD present significant challenges within a prison setting, not least because of their increased incidence; within the UK male prison estate in 2010 there was an average of 178 incidences of NSSI per 1,000 prisoners (Ministry of Justice, 2011). Despite this, research on BPD in males has been somewhat neglected, perhaps because it is more commonly associated with being female. However, in a large community study almost 35,000 adults, there is evidence that BPD is equally prevalent among men and women (Grant et al. 2008) and a recent review - although not systematic - of gender patterns in BPD argues that a previous gender bias in clinical diagnosis along with a research sampling bias accounts for inaccurate past evidence of a higher prevalence of females (Sansone \& Sansone, 2011). The review also concluded that men who suffer from BPD typically differ very little from women in levels of emotional distress, clinical presentation and self-injury; men however, use pharmacotherapy and psychotherapy services less frequently and present with different Axis I and II comorbidity, especially antisocial personality disorder; the latter often accounts for their presence in prisons, and the reluctance to seek appropriate treatment is especially worrying. While more research is needed to gain a complete and accurate picture of each of these issues, this review highlights something important: men suffer as much as women with BPD. Taking the above issues into account, we argue that there is a pressing need to understand and treat males suffering from BPD.

Therapeutic approaches to directly treat BPD, such as Dialectical Behaviour Therapy (DBT; Linehan, 1993) can be demanding on resources. However, treatments targeting the factors which may mediate the relationship between dysfunctional personality traits and problematic behaviours such as NSSI may require fewer resources because they focus on 
addressing specific psychological processes, rather than attempting to treat the underlying personality traits. Previous studies have identified mediating factors such as coping styles when trying to understand the mechanism through which personality disorders influence psychological distress such as anxiety or depression (e.g., Ireland, Brown \& Ballarini, 2006), and there is evidence to support a similar model of behavioural distress or dysregulation. Specifically, rumination - a cognitive emotion regulation or coping strategy - has been found to act as a mechanism through which BPD exerts its influence on behavioural dysregulation (Selby, Anestis, Bender \& Joiner, 2009).

Rumination has been empirically related to BPD in multiple studies (e.g., Abela, Payne \& Moussaly, 2003; Smith, Grandin, Alloy \& Abramson, 2006), and like BPD, it is associated with suicidal behaviour (Morrison and O’Connor, 2008) and NSSI (Selby, Franklin, Carson-Wing \& Rizvi, 2013). Rumination is a key feature of the emotional cascade model (Selby \& Joiner, 2009), a theoretical model that attempts to explain the relationship between emotion dysregulation in the form of BPD, and behavioural dysregulation. The model proposes that intense rumination leads to 'emotional cascades', cycles in which rumination increases the intensity of negative emotion, leading to more attention being paid to the emotion, and progressively leading to more intense rumination. According to the model, during an emotional cascade individuals may seek out a distraction in order to interfere with this cycle and obtain emotional relief. Such distracting behaviors may manifest in the many dysregulated behaviours displayed by individuals suffering from BPD. Empirical testing of this model has provided evidence for the potential role of rumination in mediating the relationship between BPD and dysregulated behaviours in student and community populations, including binge eating, drinking, non-suicidal self-injury, suicidality and suicide attempts, substance use, reckless driving, insulting someone, physical fights, and seeking 
reassurance in interpersonal relationships (Selby, Anestis, Bender, \& Joiner, 2009; Selby \& Joiner, 2013). What is not yet clear, is whether the model can be applied to forensic samples.

Prison settings can be highly stressful (especially interpersonally) making huge demands on coping resources and providing a number of opportunities for emotion dysregulation. Poor coping skills makes some prisoners with personality disorder traits vulnerable to psychological distress (Ireland et al., 2006), and there is evidence that ineffective coping plays an important role in self-injury in prisoners (see Dear, 2008, for discussion), and suicidality (e.g., Horesh et al., 1996). The prison environment is also restrictive in terms of attachment needs not being met; this is crucial to those with BPD (e.g., Agrawal, Gunderson, Holmes, \& Lyons-Ruth, 2004) and may trigger rumination and an emotional cascade. Prisoners may also be especially vulnerable to rumination because of the large amount of time available to think about one's problems and oneself; and for male offenders who have committed particularly violent crimes, there is evidence of distressing ruminations related to the assault (Evans, Ehlers, Mezey, \& Clark, 2007). Taken together, prisoners are especially vulnerable to emotional cascades and subsequent behavioural dysregulation in an attempt to cope and divert attention away from this rumination-inducing negative affect.

Theoretically, there is no reason to expect the relationships between BPD, rumination, and self-injurious behaviors to be different within forensic settings. However, because forensic settings are restrictive, behaviours that may typically be used to stop rumination and an emotional cascade may be unavailable (e.g. substances to abuse, food to binge eat). Thus, when considering how the emotional cascades model would apply to a forensic population, this reasoning should govern the choice of dysregulated behaviours, alongside the knowledge that a main priority for prisons is to reduce the highly prevalent and difficult to manage behaviours of suicide and self-injury. Thus, within the prison setting we expected the 
emotional cascades model of BPD would apply well to self-injury and suicidality. Both could potentially reduce negative affect through redirecting attention. If this is supported, this would provide valuable information as to whether rumination is a potential mediating variable that would be a meaningful target for intervention with this population. Evidence from a study using depressed patients in a non-custodial setting suggests that brief 8-week interventions that target rumination produce positive outcomes (Ramel, Goldin, Carmona, \& McQuaid, 2004); interventions targeting rumination may therefore provide an economical and effective means of addressing challenging behaviours within custodial settings.

\section{Study Rationale and Aims}

The primary aim of the study was to provide a cross-sectional analysis of the role of rumination as a mediator between BPD traits and self-injurious behaviours, testing the application of the emotional cascades model to a sample of adult male offenders. Previous studies into the model have used students and community participants (Selby et al, 2008; Selby et. al, 2009; Selby \& Joiner, 2013), and therefore it is not clear if this theoretical model can be generalised to a forensic population. The study focused on two dysregulated behaviors that are particularly relevant to the prison population: NSSI and suicidality. Examining these dysregulated behaviours in forensic setting is important because elevated rates of both dysregulated behaviours and BPD traits are typically found (Black et al., 2007), stressors are magnified in forensic settings, and at a clinical level behaviours such as self-injury can be very problematic for both the forensic population and those working with them.

This study also had a secondary aim: although past empirical tests of the emotional cascades model have used validated psychometric self-report measures to assess behavioural dysregulation, it is important to corroborate the results from these measures. While "multimethod” assessment is the gold standard (e.g., self-report measures alongside observational measures and prison reports), we also aimed in this study to determine whether psychometric 
self-report assessments of NSSI and suicidality were related to similar screening tools routinely used by the prison services. “Assessment, Care in Custody, and Teamwork” was initiated within UK Prison Services as a case management approach to detect and help prisoners at risk of suicide and/or self-injury. If prisoner's self-reports of being on an ACCT plan are related to our psychometric measures of NSSI, suicidality and BPD, the findings would suggest that these aspects of behavioural dysregulation associated with BPD traits are manifested within a prison setting.

\section{Method}

\section{Participants}

Participants were 179 adult male offenders being detained in Category C (mediumsecure) prisons in the United Kingdom. A total of 486 questionnaires were distributed overall, with a response rate of $41.6 \%$. Of these, 23 had only partially completed the questionnaire pack, and were thus removed from the study producing a final $\mathrm{N}$ of 179 . Participants were serving a sentence for a range of offences (see Table 1). The mean age of prisoners was 37.70 ( $S D=13.53$, Range 21-77). Participants were recruited during a period of lock down when prisoners were confined to their cells. Sentence length was as follows: $<12$ months (3.4\%), 1-3 years (18.8\%), 3-5 years (26.7\%), 5-10 years $(22.7 \%),>10$ years (4.0\%), and indeterminate (24.4\%). Offenders were serving sentences for the following types of offences: sexual (35.5\%), violent (non-fatal) (15.1\%), murder/manslaughter (5.8\%), possession of drugs (9.9\%), acquisitive (26.7\%), arson/attempted arson (4.7\%), fraud (1.7\%) and other (0.6\%).

\section{Measures}

One questionnaire booklet comprising several measures and additional questions was distributed to each participant. The additional questions gathered (1) demographic 
information about the participant, and (2) information regarding whether they had ever spent being monitored on a prison ACCT plan for risk of self-injury or suicide (no/yes).

\section{Identification of borderline personality traits}

Personality Diagnostic Questionnaire $4^{\text {th }}$ Edition (PDQ-4-BPD; Hyler, 1994). The PDQ is a self-report true/false questionnaire that screens for personality disorder traits or symptoms based on the DSM-IV criteria (APA, 1995). Only the 9 items from the BPD scale were used. Scores on this measure were kept continuous for some analyses; other analyses used the cutoff points recommended in the PDQ-4-BPD to define the presence or not of a significant number of BPD traits. Internal consistency (Cronbach’s alpha) in this sample was .71.

\section{Rumination}

Cognitive Emotion Regulation Questionnaire (CERQ; Garnefski, Kraaij \& Spinhoven, 2001), Focus on Thought/Rumination and Catastrophising Subscales. This questionnaire measures a number of cognitive emotion regulation strategies that may be used by an individual when experiencing negative affect. The focus on thought/rumination subscale (4 items) measures the extent to which an individual thinks about the feelings and thoughts associated with negative events. The catastrophising subscale (4 items) refers to thoughts which explicitly emphasise the terror of an experience. Participants are asked to rate each item on a 5-point Likert scale ranging from 1 (almost never) to 5 (almost always). The scales have shown reliability (most alphas exceeding .80) and test-retest reliabilities were found to be adequate to good (.41-.59) (Garnefski, Kraaij \& Spinhoven, 2001). Internal consistencies in this sample were .79 (focus on thought/rumination) and .87 (catastrophising).

Anger Rumination Scale (ARS; Sukhodolsky, Golub \& Cromwell, 2001). This scale (19 items) measures the tendency to think about current anger-provoking situations and to recall anger episodes from the past. Participants are asked to rate each item on a 4-point Likert-type scale (from $1=$ almost never to $4=$ almost always). This scale has good internal 
consistency and adequate test-retest reliability (Sukhodolsky, Golub \& Cromwell, 2001). Internal consistency in this sample was .96.

Ruminative Responses Scale (RRS), Brooding subscale from the Response Styles Questionnaire (RSQ; Nolen-Hoeksema \& Morrow, 1991). This subscale (5 items) measures depressive rumination and assesses the tendency of an individual to think about the reasons for his or her distress. Participants are asked to rate each item on a 4-point Likert-type scale $(1=$ almost never to $4=$ almost always $)$. There is adequate internal and test-retest reliability (Treynor, Gonzalez, \& Nolen-Hoeksema, 2003). Internal consistency in this sample was .90.

\section{Self-Injurious Behaviour}

NSSI: Inventory of Statements about Self-Injury (ISAS; Klonsky \& Glenn, 2008).This is a well-validated questionnaire comprised of two sections which measure (1) the frequency of NSSI, and (2) the 13 functions of NSSI, on a 3-point Likert-type scale $(0=$ not relevant at all, to 2 = very relevant). Both sections were administered but only results from the first were used for analysis within this study. The frequency of NSSI was calculated by summing the frequencies reported for each type of NSSI. Thus, it is a measure of the number of times the individual has engaged in NSSI, regardless of the method used as we wanted to capture the full range of NSSI behaviours (clinically severe and less severe). The ISAS is a reliable and valid measure of NSSI (Klonsky \& Glenn, 2008). Internal consistency in this sample was .95.

Suicidal behaviours: The Suicide Behaviours Questionnaire-Revised (SBQ-R; Osman, Bagge, Guitierrez, Konick, Kooper \& Barrios, 2001). This brief screening measure includes 4 items, each measuring a different dimension of suicidality: (1) lifetime suicide ideation and/or suicide attempt, (2) frequency of suicidal ideation over the past twelve months, (3) previous threats of a suicide attempt, and (4) self-reported likelihood of suicidal behaviour in the future. Participants are asked to endorse one option from a number of options measuring each of the four dimensions. This measure has acceptable internal consistency reliability 
estimates (Osman, et. al, 2001). Following a pilot study item 4 was removed in this study due to concerns about participants disclosing that they were likely to attempt suicide in the future within the context of a custodial environment. In sum, the measure assesses risk of suicide through self-reported suicidal ideation/behaviours, threats and attempts; as such, it is a measure of suicidality. Internal consistency in this sample was .72.

\section{Procedure}

The study was ethically approved by the University of Central Lancashire Ethics Committee and reviewed and an application to undertake research in Her Majesty’s Prison Service was also approved by the Prison Service. All questionnaires were administered to participants during a period of "lock-down” when prisoners were confined to their cells. All questionnaires were distributed and completed over one lunchtime period of approximately 2 hours, and so all persons remained in the same location for the duration of the questionnaire completion period. Participants were approached by the researcher who knocked on the cell door and provided a brief verbal explanation of the research, and if they were interested they were given the questionnaire. It was also explained that if more time or assistance with reading/writing was needed we could arrange for someone to see them on the wing. Informed consent to participate was implied through reading of the study information, completion of the questionnaire materials and subsequent return of the questionnaire pack to the researcher at an agreed time, or to a box kept in a secure location on the wing.

\section{Data analytic strategy}

Structural Equation Modeling (SEM) was used to explore the emotional cascades model, using the statistical program AMOS (Arbuckle, 2010). The maximum likelihood chisquare $\left(\chi^{2}\right)$ statistic was used to evaluate both the measurement and structural models. As the chi-square value is affected by sample size this value is not typically used alone to assess the fit of the data (Schreiber, 2008), therefore several fit indices were used: the comparative fit 
index (CFI), Tucker-Lewis index (TLI) and the root mean square error of approximation (RMSEA). Cut off levels for a good fit were taken from a review by Schreiber (2008) and were CFI > .95, TLI > .95 and RMSEA <.06-.08. Standardised regression weights were used to interpret the direct effects. The bias-corrected bootstrap confidence intervals technique was used to test the significance of standardized indirect effects (Cheung, 2009; MacKinnon, Lockwood, \&Williams, 2004). The RMediation programme (previously “PRODCLIN”; MacKinnon, Fritz, Williams \& Lockwood, 2007) was used to confirm the mediational effect; this method uses the distribution-of-product method to build confidence intervals for the mediational effects and has been shown to produce more accurate Type I error rates and good statistical power (Tofighi \& MacKinnon, 2011). As difference between models can appear small on the basis of other fit indexes (Schreiber, 2008), the AIC and BIC indexes were used to compare alternative models that were tested.

The hypothesised model was based on the model proposed by Selby et al. (2009) and included two latent variables. The first latent variable, rumination was made up of the following indicators: CERQ Rumination, CERQ Catastrophising, the Brooding subscale of the RSQ, and the total score of the ARS to measure anger rumination. The second latent variable was self-injurious behaviour to represent behavioural dysregulation and was made up of the SBQ-R total score (Suicidality) and ISAS frequency total (NSSI). The model also included the observed variable of the PDQ-4 score for BPD as the source variable in the mediational model. This model was recursive and as such was an identified model.

\section{Results}

\section{Preliminary analyses}

Prior to analyses, the data were examined for missing data at the item level, and none contained more than 5.5\% missing data. Missing items were estimated using person mean substitution (PMS) (as recommended by Hawthorne and Elliot, 2005, with this amount of 
missing data), where the mean score of the other items on a particular subscale of a questionnaire for a particular case was inserted. There were no major deviations from normality with the exception of the ISAS total frequency of self-injury variable ( $\mathrm{S}=6.12, \mathrm{~K}$ = 43.85); reciprocal transformation (Field, 2009) was used to address this and reduced skewness and kurtosis $(\mathrm{S}=1.43, \mathrm{~K}=.24)$. There were no extreme univariate outliers within the other variables and these variables showed only mild-moderate levels of skewness (range .263 to 1.280 ) and kurtosis (range -.716 to .653 ).

Of the 179 offenders, 42 (23.5\%) had engaged in lifetime NSSI. The median number of incidents across all methods of NSSI was 8.5 (mode $=1$, minimum $=1$, maximum $=$ 4,000). Similar to other studies of personality disordered offenders (e.g., Mannion, 2009), the most frequently endorsed method was cutting (59.5\%). Regarding suicidality, the mean score was $4.89(S D=2.50$; Table 2$)$, with $50.3 \%$ of the sample indicating at least some level of suicidality.

Demographic characteristics of the sample and their association with BPD trait status (low or high) are shown in Table. 1. A total of 32 offenders (17.9\%) met the PDQ-4 criteria for BPD traits. This was lower than the prevalence found in previous studies of male prisoners (e.g. 26.8\%, Black, 2007; 25-50\%, Sansone \& Sansone, 2009).

\section{Measurement Model}

The correlations between all variables within the hypothesised model are shown in Table 2. Prior to analysing the SEM model, the measurement model was examined in order to assess the appropriateness of the latent variables (Kline, 2011). Analysis of the measurement model indicated that the two latent variables (rumination and self-injurious behaviour), were correlated with each other. Model fit indices indicated that this model did not fit the data well: $\chi^{2}(8)=30.366, p<.001 ; \mathrm{CFI}=.947 ; \mathrm{TLI}=.900 ;$ RMSEA $=.125$. The results suggested that correlating the error variances associated with the CERQ Rumination and CERQ 
Catastrophising variables would improve model fit. This path was added and with this adjustment the model was an excellent fit to the data $\left(\chi^{2}(7)=8.476, p>.05\right.$; CFI $=.996$; TLI $=.992 ;$ RMSEA = .034). All standardized factor loadings between the measured variables and their respective latent variables were significant $(p<.001)$ and ranged from .63 to .86 . The correlation between the two latent variables was $r=.67, p<.001$.

$>>$ insert tables 1 and $2<<$

\section{Structural model analyses}

The hypothesised structural model (Model 1) is shown in Figure 1. Standardised direct and indirect effects are reported for all models; all path coefficients for the direct effects are displayed in Figure 1, while the significance of the indirect effects were based on both bias-corrected bootstrap and distribution-of-product method confidence intervals and are reported below. Model fit indices indicated that the model fit the data well $\left(\chi^{2}(11)=20.389\right.$, $p<.05 ; \mathrm{CFI}=.983 ; \mathrm{TLI}=.968 ; \mathrm{RMSEA}=.069)$. Consistent with the findings of Selby et al., (2009), the direct path from BPD to rumination was significant $(\beta=.735, \mathrm{p}<.001)$, as was the direct path from rumination to self-injurious behavior $\beta=.344, p<.05$ ), and from BPD to selfinjurious behaviour $(\beta=.433, p<.001)$. The indirect path from BPD to self-injurious behaviour with rumination as the mediator was also significant (standardized indirect effect $=$ .253 , bias-corrected bootstrap lower $\mathrm{CI}=.050$ and upper $\mathrm{CI}=.482, p<.05$ ). The RMediation programme (MacKinnon et al., 2004) was used to further test this mediational effect using the distribution-of-product method. The unstandardised coefficients and standard errors of the a and $\mathrm{b}$ paths between BPD and rumination and between rumination and suicidal behaviour, were entered into RMediation to yield lower and upper 95\% confidence limits of .046 and .378, thus a significant indirect effect. In sum, the results were consistent with that of a partial 
mediation effect, given a significant direct path between BPD and self-injurious behaviour in the presence of a significant indirect effect.

To address the possibility that the associations between BPD and the self-injurious behaviour latent were an artefact of criterion overlap due i.e., due to the inclusion of a selfinjury/suicide item within the BPD construct, we followed Selby et al., (2009) and removed this item and respecified the model. Having removed this item from the BPD construct, the direct path from BPD to rumination remained significant $(\beta=.732, p<.001)$, as did the direct path from rumination to self-injurious behaviour $(\beta=.576, p<.001)$, while the direct path from BPD to self-injurious behaviour became non-significant $(\beta=.116, p>.05)$. However, there remained a significant indirect effect of BPD on self-injurious behaviour through rumination (standardized indirect effect $=.422$, bias-corrected bootstrap lower CI $=.189$, upper $\mathrm{CI}=.615, p<.05$ ). This pattern of results suggests that with the self-injury criterion removed from BPD, rumination fully mediates the relationship between BPD traits and selfinjurious behaviour, as was found by Selby et al. (2009). This model was also a good fit to the data $\left(\chi^{2}(11)=23.077, \mathrm{p}<.05 ;\right.$ CFI $=.978 ;$ TLI $=.957 ;$ RMSEA $\left.=.079\right)$. RMediation yielded lower and upper 95\% confidence limits of .188 and .612, and thus a significant indirect effect. In sum, the results show that the residual BPD construct with the selfinjury/suicide item excluded still results in a mediational effect on self-injurious behaviour. In this second model the results were consistent with a full mediational effect, given the nonsignificant direct path between BPD and self-injurious behaviour in the presence of a significant indirect effect. That the direct effect between BPD and self-injurious behaviour was non-significant in this model but significant in the first, suggests that this relationship was influenced by criterion overlap. Importantly though, the mediational effect was still present in this second model. 
AIC was used to examine differences in model fit. Analysis of the two models indicated that the first model (AIC $=54.389$ ) was a marginally better fit than the second (AIC = 57.077; Burnham \& Anderson, 2004).

>>insert Figure $1<<<$

\section{Additional Analyses to Explore BPD and Self-Reported Custodial Behaviour}

Analyses were conducted across the whole sample to explore whether self-reported prison behaviour (ACCT status) correlated with the psychometric measures used to assess NSSI and suicidality. Both measures were significantly correlated with ACCT status: NSSI: $r$ $=.41, p<.01$; suicidality: $r=.49, \mathrm{p}<.01$ ), indicating that these measures did reflect selfreports of actual prison behaviour. Notably, none of the measures of rumination were significantly correlated with ACCT status. Additional analyses showed that those with high BPD traits were significantly more likely to self-report having previously spent time being monitored for risk of NSSI and/or suicide within the prison (ACCT status; Table. 1).

\section{Discussion}

The results of this study provide evidence to suggest that the emotional cascade model is applicable to BPD traits in a forensic setting with adult male offenders, thus extending the small number of studies that have empirically tested the model. Evidence was found for the role of rumination in predicting broadly defined self-injurious behavior, consisting of both non-suicidal self-injury and suicidality. These findings are consistent with other empirical tests of the model (e.g., Selby et al. 2009), and can potentially improve our understanding of dysregulated behavior in forensic settings.

The findings also indicated in the structural model investigated that the association between BPD and dysregulated behaviour was fully mediated by rumination, after accounting 
for the criterion overlap of the BPD self-injury/suicide item and the dysregulated behaviors latent variable. This suggests that rumination may be a particularly important contributing factor to dysregulated behaviors among those with elevated BPD traits, and it has the potential to serve as a clear and concrete issue to address in clinical forensic settings.

Although our interest was in the indirect effect of BPD on self-injurious behaviour, the fact that the direct relationship between BPD and self-injurious behaviour did not persist once the self-injury/suicide item was removed from the BPD construct, suggests this particular relationship may have been influenced by criterion overlap. However, it must be noted that criterion or "item trimming" alters the conceptual meaning of the BPD construct through the removal of theoretically importance variance. Thus, from these results alone we cannot discount the presence of a direct relationship between BPD and self-injurious behaviour in this population (beyond the contributions of rumination). Future research will need to replicate these findings using multi-method assessments of BPD and self-injurious behaviour, as this would be a more powerful test of whether conceptual overlap is causing spurious relationships because common method variance is avoided. Encouragingly, our pattern of results is wholly consistent with Selby et al.'s. (2009) empirical test of the model within a student population.

Theoretically, these findings are informative for understanding NSSI and suicidality in this population. According to our findings, rumination is a mechanism through which prisoners with BPD traits may engage in NSSI or suicidal behaviours/threats (or experience suicidal cognitions). Rumination may serve to intensify and prolong negative affect and act as a mechanism for both dysregulated behaviours regardless of population type (i.e., prisoners in this study, or student populations in previous research). There may be other important cognitive mechanisms (e.g., coping styles), but prison settings are an especially fertile ground 
for rumination as there can be too much time to think, especially during the evenings which can be isolating while prisoners reside in their cells.

The exact nature of this rumination is broad and needs further investigation, but our findings suggest it at least includes general ruminative thinking and catastrophizing, but also more specific depressive ruminations about one's distress and ruminations about current or past angry episodes. Consistent with Selby et al., (2009), the latter two were most strongly related to the latent variable of rumination in this study. Given that negative affect/emotional distress and anger are hallmarks of BPD (Sauer \& Baer, 2012), ruminations could center largely around these, and there is indeed evidence to suggest that both depression and anger rumination are highly related to BPD (e.g., Baer \& Sauer, 2011). Because such rumination intensifies negative affect, the implications of anger rumination could be especially difficult to deal with within the prison environment: given the potential repercussions of violence within the prison it would not be surprising that some individuals suppress their anger and instead regulate their negative affect through NSSI or suicidal behaviour. These behaviours may serve an important function in directing attention away from the negative affect that is induced by ruminative thought.

What is not understood from our research is the specific content of these ruminations, and this could be especially important in offender populations and also in different types of offenders. In violent offenders - of which there were many in this sample - the content of ruminations could easily relate to an anger source; this could be the previously committed violent crime which could be triggered by intrusive memories of the event (Evans et al., 2007), a current situation that has angered them, or ruminations about a provocation. Thus, there may be a moderating effect of offender type such that in the emotional cascades model violent or anti-social personality traits exacerbate anger rumination in individuals with BPD 
traits. Future research should explore whether such offender characteristics moderate emotional cascades, alongside a moderating or mediating role of rumination content.

Note that previous empirical tests of the emotional cascades model have approached the issue of comorbid personality disorders from a specificity angle: statistical control of Cluster B personality disorders within the emotional cascades model has enabled the assessment of specificity of the model to BPD (e.g., Selby et al., 2009; Selby \& Joiner, 2013). However, while the findings have supported the validity of the model, in many cases this can be an overly stringent approach to testing due to the removal of real construct variance from BPD (see Miller \& Chapman, 2001, for discussion of this issue), especially when assessing BPD traits or subclinical levels of the disorder as in this study.

In relation to the second aim of the study, the results suggest that psychometric measures used within this study reflect self-reports of prison behaviour in terms of whether or not the individual had previously spent time being monitored in the prison for risk of suicide or NSSI (ACCT status). Moreover, we found that high BPD traits was related to having being monitored on an ACCT plan before, suggesting that behavioural dysregulation in the form of self-injury/suicidality associated with BPD is manifested within a prison setting. However, while not an objective of this study, it is important to highlight that none of rumination measures were significantly correlated with ACCT status. These findings indicate that those individuals who are identified within the prison system as being at risk of NSSI or suicide are not characterised by a tendency to ruminate. However, these findings could reflect the trait rather than state rumination measure that was used, or alternatively that the process of rumination is more important in understanding longer-term NSSI. Regarding the latter, both of the psychometric measures of self-injurious behaviour ask the individual to consider incidences of this over their lifetime, whereas ACCT status relates to only the period of imprisonment, and could therefore reflect more recent behaviour. This further highlights the 
importance of multi-method assessment in future empirical tests of the emotional cascades model.

The results of this study should be interpreted within the context of several limitations. In addition to those noted above, the cross-sectional design of the study was necessary given the constraints of collecting data within the prison environment, but this limited the extent to which the emotional cascades model could be tested. In order to show that rumination exacerbates negative affect, which is subsequently interrupted by dysregulated behavior, a longitudinal design is necessary. Selby et al. (2013) adopted this design in a recent study using experiencing sampling methods, with results provided supporting for the validity of the model. A further limitation relates to the overall and subgroup sample sizes, which were relatively small. This could limit the extent to which these findings could be generalised across a similar sample, but our findings are consistent with the emotional cascades model despite a small size, providing support for the robustness of the model.

As we have noted, common method variance is an issue in any study that solely utilizes self-report methodology, but there are also other problems with self-report measures such as social desirability. However, social desirability is not always a response bias that attenuates criterion validity (Ones, Visweswaran, \& Reiss, 1996), and nor are self-report measures of BPD any less valid than diagnostic interviews because the former are effective in assessing experiential BPD symptoms (Hopwood et al., 2008) (thus assessing “BPD traits” rather than a "BPD diagnosis”). Hopwood et al. advocate a combined approach to BPD assessment and we thus recommend that future research replicate our findings while supplementing self-report measures with other such corroborating sources such as diagnostic interviews of BPD and behavioural assessments of self-injurious behaviours. This is perhaps more important in offender samples where special care must be taken when selecting and 
administering assessment measures because the accuracy of these measures can be compromised through unique characteristics of the environment and sample (see Megargee, 1995, for discussion). In particular, the prison setting can impact on the measurement of selfinjurious behaviour because prisoners may be less likely to report engaging in such behaviour, or may actually be less able to engage in this behaviour due to the practical constraints of the environment (e.g., staff checks) and constraints on the expression of emotions.

In addition to this, in this study we wanted to capture the full range of NSSI behaviours (clinically severe such as cutting and less severe such as biting), but future research should apply the emotional cascades model to NSSI behaviours of varying degrees of severity given evidence of clinically-distinct profiles for such NSSI groups (e.g., Klonsky \& Olino, 2008; Whitlock, Muehlenkamp \& Eckenrode’ 2008). Finally, research will need to determine whether the emotional cascades model applies to other dysregulated behaviours that are accessible within the prison environment (e.g., aggression), consider participant factors (e.g., ethnicity) that are important determinants of suicidality and which may shape the findings (Selby \& Joiner, 2008), and assess application of the model to female offenders.

This study highlights a number of potential clinical implications that should be considered. The prevalence rate of BPD traits (17.9\%) within this sample, combined with the finding that individuals with high BPD traits were significantly more likely to have been placed on monitoring for risk of NSSI or suicide, highlights the benefits of identifying and treating offenders with BPD traits. The findings regarding the role that rumination plays in mediating the relationship between BPD and self-injurious behaviours provides some promising ideas for managing this risk within custodial settings. Treatment for BPD can be resource-intensive; the outcome of this study indicates that treatment approaches aimed at enabling offenders to reduce the degree to which they ruminate may be effective in reducing 
risk of NSSI and suicidality. Clearly, these findings are only the first step in building an evidence base to support this theory.

\section{References}

Abela, J. R. Z., Payne, A. V. L., \& Moussaly, N. (2003). Cognitive vulnerability to depression in individuals with borderline personality disorder. Journal of Personality Disorders, 17 (4), 319-329.

Agrawal, H.R., Gunderson, J., Holmes, B.M., \& Lyons-Ruth, K. (2004). Attachment studies with borderline patients: A review. Harvard Review of Psychiatry, 2, 2, 94-104.

American Psychiatric Association. (1995). Diagnostic and statistical manual of mental disorders: DSM-IV (4th ed.). Washington, DC: American Psychiatric Press.

Arbuckle, J. L. (2010). AMOS 19.0 (Computer software). Chicago: SPSS.

Baer, R. A., \& Sauer, E. (2011). Relationships between depressive rumination, anger rumination, and borderline personality features. Personality Disorders: Theory, Research, and Treatment, 2, 2, 142-150.

Black, D.W., Gunter, T., Allen, J., Blukm, N., Arndt, S., Wenman, G., \&Sielneni, B. (2007). Borderline personality disorder in male and female offenders newly committed to prison. Comprehensive Psychiatry, 48, 400-405.

Bornovalova, M.A., Gratz, K.L., Daughters, S.B., Nick, B., Delayny-Brumsey, A., Lynch, T.R., Kosson, D., \& Lejuez, C.W. (2008). A multimodal assessment of the relationship between emotion dysregulation and borderline personality disorder among inner-city substance users in residential treatment. Journal of Psychiatric Research, 42, 9, 717-26.

Burnham, K. P., \& Anderson, D. R. (2004). Multimodel inference: Understanding AIC and BIC in model selection. Sociological Methods and Research, 33, 2, 261-304. 
Cheung, M. W-L. (2009) Comparison of methods for constructing confidence intervals of standardized indirect effects. Behavior Research Methods, 41, 2, 425-438.

Conklin, C.Z., Bradley, R., \&Westen, D. (2006). Affect regulation in borderline personality disorder. The Journal of Nervous and Mental Disease, 194, 2, 69-77.

Evans, C., Ehlers, A., Mezey, G., \& Clark, D. M. (2007). Intrusive memories and ruminations related to violent crime among young offenders: Phenomenological characteristics

Field, A. (2009). Discovering statistics using SPSS. Third Edition. Sage Publications.

Dear, G. E. (2008). Ten Years of Research into Self-Harm in the Western Australian Prison System: Where to Next? Psychiatry, Psychology and Law, 15, 3, 469-481

Gardner, K. J., Archer, J., \& Jackson. S. (2012). Does maladaptive coping mediate the relationship between borderline personality traits and reactive and proactive aggression? Aggressive Behaviour, 38, 403-413.

Garnefski, N., Kraaij, V., \& Spinhoven, P. (2001). Negative life events, cognitive emotional regulation and emotional problems. Personality and Individual Differences, 30, 13111327.

Grant, B.F., Chou, S.P., Goldstein, R.B., Huang, B., Stinson, F.S., Saha, T.D., Smith, S.M., Dawson, D. A., Pulay, A. J., Pickering, R. P., \& Ruan, W.J. (2008). Prevalence, correlates, disability, and comorbidity of DSM-IV borderline personality disorder: Results from the Wave 2 National Epidemiologic Survey on Alcohol and Related Conditions. Journal of Clinical Psychiatry, 69, 4, 533-545.

Hawton, K., Linsell, L., Adeniji, T., Sariaslan, A., \& Fazel, S. (2014). Self-harm in prisons in England and Wales: An epidemiological study of prevalence, risk factors, clustering, and subsequent suicide. The Lancet, 383, 29, 1147-1154.

Hawthorne, G. \& Elliott, P. (2005). Imputing cross-sectional missing data: Comparison of common techniques. Australian and New Zealand Journal of Psychiatry, 39, 583-590. 
Horesh, N., Rolnick, T., Iancu, I., Dannon, P., Lepkifker, E., Apter, A., \& Kotler, M. (1996). Coping styles and suicide risk. Acta Psychiatrica Scandinavica, 93, 6, 489-493.

Hopwood, C.J., Morey, L., Edelen, M.O., Shea, M.T., Grilo, C.M., Sanislow, C.A., McGlashan, T.H., Daversa, M.T., Gunderson, J.G., Zanarini, M., Markowitz, J.C., Skodol, A.E., (2008). A comparison of interview and self-report methods for the assessment of borderline personality disorder criteria. Psychological Assessment 20, $81-85$.

Hyler, S. E. (1994). Personality Diagnostic Questionnaire-4. New York: New York State Psychiatric Institute.

Ireland, J. L., Brown, S.L., \& Ballarini, S. (2006). Maladaptive personality traits, coping styles and psychological distress: A study of adult male prisoners. Personality and Individual Differences, 41, 561-573.

Klonsky, D. E., Oltmanns, T. F., \& Turkheimer, E. (2003). Deliberate self-harm in a nonclinical population: prevalence and psychological correlates. American Journal of Psychiatry, 160, 1501-1508.

Klonsky, D. E., \& Glenn, C. R. (2008). Assessing the functions of non-suicidal self-injury: Psychometric properties of the Inventory of Statements About Self-Injury (ISAS). Journal of Psychopathology and Behaviour Assessment, 31, 215-219.

Klonsky, D.E., \& Olino, T. M. (2008). Identifying clinically distinct subgroups of selfinjurers among young adults: A latent class analysis. Journal of Consulting and Clinical Psychology, 76, 1, 22-27.

Eline, R. B. (2011). Principles and practice of structural equation modeling ( ${ }^{\text {rd }}$ ed.). Guilford Press: New York.

Leue, A., Borchard, B., \& Hoyer, J. (2004). Mental disorders in a forensic sample of sexual offenders. European Psychiatry, 19, 3, 123-130. 
Linehan, M. (1993). Cognitive-behavioral treatment of borderline personality disorder. New York: The Guildford Press.

MacKinnon, D. P., Lockwood, C. M., \&Williams, J. (2004). Confidence limits for the indirect effect: Distribution of the product and resampling methods. Multivariate Behavior Research, 39, 99-128.

MacKinnon, D. P., Fritz, M. S., Williams, J., \& Lockwood, C. M. (2007). Distribution of the product confidence limits for the indirect effect: Program PRODCLIN. Behavior Research Methods, 39, 384-389.

Mannion, A. (2009). Self-harm in a dangerous and severely personality disordered population. The Journal of Forensic Psychiatry \& Psychology, 20, 2, 322-331

Megargee, 1995. Assessment research in correctional settings: Methodological issues and practical problems. Psychological Assessment, 7, 3, 359-366.

Miller, G. A., \& Chapman, J. P. (2001). Misunderstanding Analysis of Covariance. Journal of Abnormal Psychology, 110, 1, 40-48.

Ministry of Justice England and Wales. (2011). Safety in Custody 2010 Statistics bulletin.

Morrison, R., \& O'Connor, R.C. (2008). A systematic review of the relationship between rumination and suicidality. Suicide and Life-Threatening Behavior, 38, 523-538.

Ones, D. S., Viswesvaran, C., \& Reiss, A. (1996). Role of social desirability in personality testing for personnel selection: The red herring. Journal of Applied Psychology, 81, 6, 660-679.

Osman, A., Bagge, C. L., Guitierrez, P. M., Konick, L.C., Kooper, B.A., \& Barrios, F. X. (2001). The Suicidal Behaviors Questionnaire-Revised (SBQ-R): Validation with clinical and non-clinical samples. Assessment, 5, 443-454. 
Nolen-Hoeksema, S., \& Morrow, J. (1991). A prospective study of depression and posttraumatic stress symptoms after a natural disaster: The 1989 Loma Prieta earthquake. Journal of Personality and Social Psychology, 61, 115-121.

Radloff, L.S. (1977). The CES-D Scale. A self-report depression scale for research in the general population. Applied Psychological Measurement, 1, 3, 385-401.

Ramel, W., Goldin, P. R., Carmona, P. E., \& McQuaid, J. R. (2004). The effects of mindfulness meditation on cognitive processes and affect in patients with past depression. Cognitive Therapy and Research, 28,433.

Sauer, S. E., \& Baer, R. A. (2012). Ruminative and mindful self-focused attention in borderline personality disorder. Personality Disorders: Theory, Research, and Treatment, 3, 433-441.

Samuels, J., Eaton, W.W., O.J. Bienvenu III., Brown, C.H., Costa, P.T., Nestadt, G. (2002). Prevalence and correlates of personality disorders in a community sample. British Journal of Psychiatry, 180, 536-542.

Sansone, R. A., \& Sansone, L. A. (2009). Borderline personality and criminality. Psychiatry (Edgmont), 6, 10, 16-20.

Sansone, R. A., \& Sansone, L. A. (2011). Gender patterns in borderline personality disorder. Innovations in Clinical Neuroscience, 8, 5, 16-20.

Sansone, R. A., Wiederman, M. W., \& Sansone, L. A. (2000). The prevalence of borderline personality disorder among individuals with obesity. A critical review of the literature. Eating Behaviours, 1, 93-104.

Schreiber, J.B. (2008). Core reporting practices in structural equation modeling. Research in Social and Administrative Pharmacy, 4, 2, 83-97. 
Selby, E. A., Anestis, M. D., \& Joiner, T.E. (2008). Understanding the relationship between emotional and behavioural dysregulation: Emotional cascades. Behaviour Research and Therapy, 46, 593-611.

Selby, E.A., Anestis, M.D., Bender, T.W., \& Joiner, T.E. (2009). An exploration of the Emotional Cascades Model in Borderline Personality Disorder. Journal of Abnormal Psychology, 118, 375-387.

Selby, E. A., \& Joiner Jr, T. E. (2008). Ethnic variations in the structure of borderline personality disorder symptomatology. Journal of Psychiatric Research, 43, 2, 115 123.

Selby, E.A., \& Joiner, T.E. (2009). Cascades of emotion: The emergence of Borderline Personality Disorder from emotional and behavioural dysregulation, Review of General Psychology, 13, 3, 219-229.

Selby, E. A., \& Joiner, T. E., Jr. (2013). Emotional Cascades as prospective predictors of dysregulated behaviors in Borderline Personality Disorder. Personality Disorders: Theory, Research, and Treatment, 4, 168-174.

Selby, E. A., Franklin, J., Carson-Wong, A., \& Rizvi, S. L. (2013). Emotional cascades and self-injury: Investigating instability of rumination and negative emotion. Journal of Clinical Psychology, 69, 12, 1213-27.

Smith, J. M., Grandin, L. D., Alloy, L. B., \& Abramson, L. Y. (2006). Cognitive vulnerability to depression and Axis II personality dysfunction. Cognitive Therapy and Research, 30, 609-621.

Sukhodolsky, D. G., Golub, A., \& Cromwell, E. N. (2001). Development and validation of the anger rumination scale. Personality and Individual Differences, 31, 5, 689-700.

Tofighi, D. \& MacKinnon, D. P. (2011). RMediation: An R package for mediation analysis confidence intervals. Behavior Research Methods, 43, 692-700 
Tragesser, S.L., \& Robinson, J. (2009). The role of affective instability and UPPS impulsivity in borderline personality disorder. Journal of Personality Disorders, 23, 370-383.

Treynor, W., Gonzalez, R., \& Nolen-Hoeksema, S. (2003). Rumination reconsidered: A psychometric analysis. Cognitive therapy and Research, 27, 247-259

Trull, T. J., Sher, K. J., Minks-Brown, C., Durbin, J., \& Burr, R. (2000). Borderline personality disorder and substance use disorders: a review and integration. Clinical Psychology Review, 20, 2, 235-253.

Whitlock, J.L., Muehlenkamp, J., Eckenrode, J. (2008). Variation in non-suicidal self-injury: Identification of latent classes in a community population of young adults. Journal of Clinical Child and Adolescent Psychology, 37, 4, 725-735. 
Table 1:

Characteristics of Offenders with Low and High BPD Traits

\begin{tabular}{|c|c|c|c|c|}
\hline & & Low BPD traits $(n=32)$ & High BPD traits $(n=147)$ & $p$ \\
\hline \multirow[t]{6}{*}{ Length of sentence } & $<12$ months & $9.7 \%$ & $2.1 \%$ & $.023 * 1$ \\
\hline & $1-3$ years & $19.4 \%$ & $18.6 \%$ & \\
\hline & 3-5 years & $9.7 \%$ & $30.3 \%$ & \\
\hline & $5-10$ years & $22.6 \%$ & $22.8 \%$ & \\
\hline & $>10$ years & $0 \%$ & $4.8 \%$ & \\
\hline & Indeterminate (Life/IPP sentence) & $38.7 \%$ & $21.4 \%$ & \\
\hline \multirow[t]{7}{*}{ Offence type } & Sexual & $41.9 \%$ & $34.3 \%$ & $.807^{1}$ \\
\hline & Violent (non-fatal) & $19.4 \%$ & $14.3 \%$ & \\
\hline & Murder/Manslaughter & $3.2 \%$ & $6.4 \%$ & \\
\hline & Possession of drugs & $9.7 \%$ & $10.0 \%$ & \\
\hline & Acquisitive offences & $19.4 \%$ & $28.6 \%$ & \\
\hline & Arson/Attempted Arson & $6.5 \%$ & $4.3 \%$ & \\
\hline & Fraud offences & $0 \%$ & $2.1 \%$ & \\
\hline \multirow[t]{2}{*}{ Previous self-injury/suicide monitoring (ACCT) } & Yes & $50.0 \%$ & $15.2 \%$ & $.001^{* * * 1}$ \\
\hline & No & $50.0 \%$ & $84.8 \%$ & \\
\hline
\end{tabular}

Note: IPP = Imprisonment for Public Protection.

${ }^{1} p$ value obtained from Pearson chi square test. ${ }^{*} p<.05$. ${ }^{*} p<.01 .{ }^{* * *} p<.001$. 
Table 2:

Bivariate Correlations, Means and Standard Deviations for Observed Variables within the Structural Equation Model

\begin{tabular}{llllllll}
\hline & 1 & 2 & 3 & 4 & 5 & 6 & 7 \\
\hline 1. BPD & - & & & & & & \\
2. NSSI & $.48^{* * *}$ & - & & & & & \\
3. Suicidality & $.46^{* * *}$ & $.47^{* * *}$ & - & & & & \\
4. Catastrophising (CERQ) & $.47^{* * *}$ & $.24^{* * *}$ & $.28^{* * *}$ & - & & & \\
5. Rumination (CERQ) & $.38^{* * *}$ & $.20^{* *}$ & $.35^{* * *}$ & $.66^{* * *}$ & - & & \\
6. Anger rumination (ARS) & $.67^{* * *}$ & $.33^{* * *}$ & $.41^{* * *}$ & $.60^{* * *}$ & $.53^{* * *}$ & - & \\
7. Brooding (RSQ) & $.59^{* * *}$ & $.39^{* * *}$ & $.45^{* * *}$ & $.60^{* * *}$ & $.54^{* * *}$ & $.69 * * *$ & - \\
Mean & 2.80 & 90.77 & 4.89 & 8.80 & 10.55 & 34.55 & 9.85 \\
SD & 2.13 & 433.70 & 2.50 & 4.28 & 3.78 & 14.38 & 3.99 \\
Range & $0-9$ & $0-4000$ & $3-12$ & $4-20$ & $4-20$ & $19-76$ & $5-20$ \\
\hline
\end{tabular}

Note: Mean and standard deviations shown for the self-injury variable are prior to transformation. $\mathrm{CERQ}=$ Cognitive Emotion Regulation Questionnaire; $\mathrm{ARS}=$ Anger Rumination Scale; RSQ = Response Styles Questionnaire.

$*^{*} p<.05 .{ }^{* *} p<.01, * * * p<.001$ 


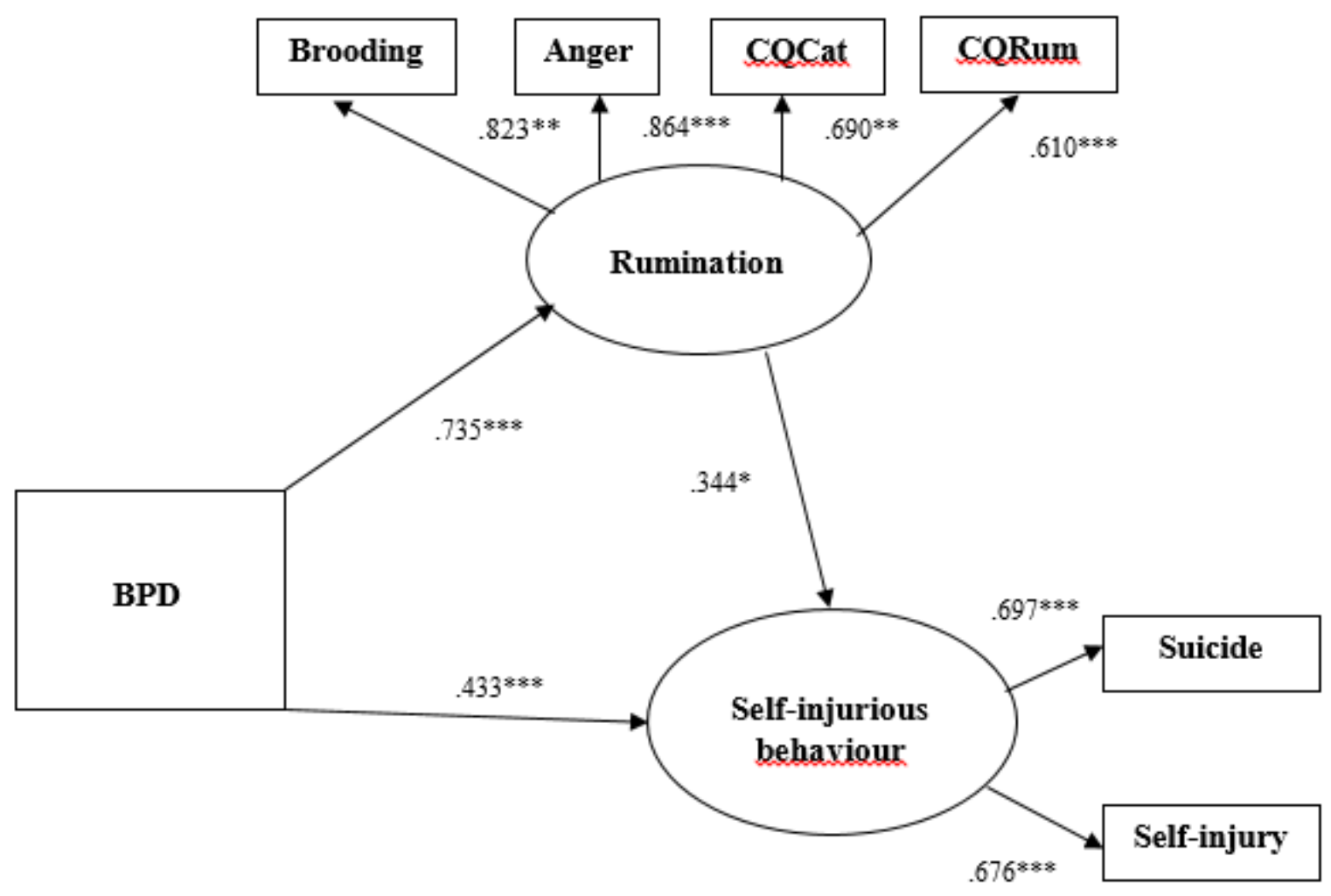

Figure 1: Structural equation model testing the mediational effect of rumination on the relationship between symptoms of BPD and self-injurious behaviour.

Note: Standardised paths shown and all are significant. To achieve identification of the models, one regression coefficient for each latent variable and all regression coefficients of the error terms over the observed endogenous variables were set at unity. CQCat = Cognitive Emotion Regulation Questionnaire Catastrophising subscale; CQRum = Cognitive Emotion Regulation Questionnaire Rumination subscale; Anger = Anger Rumination Scale; Brooding $=$ Response Styles Questionnaire Brooding subscale.

${ }^{*} p<.05 .{ }^{* *} p<.01 .{ }^{* * *} p<.001$ 